\title{
Modernidad, formación y empleo en el Marruecos de las mujeres
}

\author{
Francisco Alberto Vallejo Peña \\ Universidad de Málaga. Departamento de Derecho del Estado y Sociología \\ favallejo@uma.es
}

Recibido: 29-07-2011

Aceptado: 01-03-2012

\section{Resumen}

Marruecos vive en la actualidad un intenso proceso de cambio social que tiene a sus mujeres en el núcleo. Entre las diversas transformaciones sociales, políticas y económicas vividas en el Reino Alauí, destaca notoriamente el papel del acceso femenino a roles laborales y formativos como nunca antes se había dado. En este sentido, se observa que tanto el desempeño de labores fuera del hogar como la percepción de un salario propio está cambiando la mentalidad de la mujer y sus actuaciones en otros campos, en los que va ganando progresivamente protagonismo, tal y como confirma la revisión teórica de autoras como Chafai, Mernissi o Alberdi, entre otras.

Para precisar las consecuencias de estos cambios, se han analizado diversas variables explotadas por la Encuesta Mundial de Valores para Marruecos en 2001 y 2007, todas ellas relativas a las dimensiones de formación y empleo. Asimismo, los resultados se han contrastado con los discursos de informantes clave recogidos en trabajos de campo en Marruecos realizados para AECID por las universidades de Málaga y Abdelmalek Essaadi entre 2007 y 2008 (La identidad de género de la mujer marroquî).

Palabras clave: estudios sobre la mujer; cambio social; sociología del género; trabajo; educación.

\section{Abstract. Modernity, training and employment for Moroccan women}

Morocco is currently experiencing an intense process of social change in which women are at the core. One of the most important social, political and economic transformations occurring in the country is the access by women to jobs and training. We observe that the ability to work outside the home and earn a salary is changing women's mentality as well as their actions in other fields in which they are gradually gaining prominence, as the theoretical review of works by other authors such as Chafai, Mernissi, or Alberdi, among others, attests to.

To explore the consequences of these changes, several variables relating to the dimensions of training and employment of the World Values Survey for Morocco of 2001 and 2007 are analysed. The results are compared to the discourses of key informants collected 
in fieldwork conducted in Morocco by the universities of Malaga and Abdelmalek Essaadi from 2007 to 2008 (Gender identity of Moroccan women) for the Spanish Agency for International Development Cooperation (AECID).

Keywords: women's studies; social change; sociology of gender; labour; education.

\author{
Sumario \\ 1. Planteamiento inicial \\ 4. Empleo y formación desde \\ 2. Antecedentes teóricos \\ e investigaciones previas \\ 3. Objetivos y metodología \\ la perspectiva de la mujer marroquí: \\ presentación de resultados \\ 5. Conclusiones \\ Referencias bibliográficas
}

\title{
1. Planteamiento inicial
}

Las transformaciones sociales y políticas recientemente vividas en la sociedad marroquí han tenido a la mujer como gran protagonista. Nos encontramos ante un caso que llama particularmente la atención, tanto en el ámbito del Magreb como en el del conjunto de países musulmanes. El movimiento de la mujer avanza en el Reino Alauí a ritmo lento pero firme y ha obtenido ya los primeros resultados tangibles, que de ninguna manera deben pasar desapercibidos, dada su significación política y sociológica. El objetivo del presente trabajo es valorar la reciente evolución de Marruecos y sus mujeres en materia de formación y empleo. Esta investigación se va a realizar desde la perspectiva de los valores, considerando su evolución en el país africano desde 2001. Para su análisis, se han explotado los resultados de la Encuesta Mundial de Valores (WVS) de Marruecos en diversas variables relativas a las dimensiones sobre formación y empleo de sus hombres y mujeres. Asimismo, con los resultados de esta fase cuantitativa, se pretende contrastar y confirmar las conclusiones obtenidas tras los trabajos de campo cualitativos ejecutados en Marruecos en los ejercicios de 2007 y 2008 en dos proyectos AECID sobre identidad de género de la mujer marroquí, que presentan una particular incidencia en las dimensiones sobre la formación y el empleo. Se trata de investigaciones ejecutadas por un equipo de investigación bilateral en el que participa una universidad española en colaboración con su contraparte marroquí ${ }^{1}$. Para tal efecto, se ha realizado una selección previa de variables explicativas del fenómeno ya difundidas por la Encuesta Mundial de Valores: Ser ama de casa llena tanto como un trabajo remunerado (reacción ante la frase), Principales motivos para trabajar, Importancia del trabajo en la vida, Importancia de ir a la universidad

1. José Fernando TROYANO (coord.) et al. (2007, 2008), La identidad de género de la mujer marroquí (I y II). Programa: PCI Mediterráneo; AECID; Ministerio de Asuntos Exteriores. Entidades ejecutoras: Universidad de Málaga y Universidad Abdelmalek Essaadi. 
para hombres y mujeres (¿más para un chico que para una chica?) y La consideración del empleo como un derecho preferente para los hombres respecto a las mujeres.

A continuación, se expone una aproximación teórica basada en fuentes procedentes de las perspectivas del desarrollo, la modernidad y el cambio social; priorizando, en todos los casos, una visión de género en materia de formación y empleo. Posteriormente, se resumen los objetivos y la metodología de la investigación, para continuar con un análisis contrastado de datos cualitativos y cuantitativos (WVS 2001 y 2007) en Marruecos y finalizar con las principales conclusiones obtenidas.

\section{Antecedentes teóricos e investigaciones previas}

El nuevo Marruecos presenta unas condiciones sociales, políticas, laborales y económicas para las mujeres muy distintas a las de mediados del siglo xx. Ahora la mujer comienza a adquirir roles protagonistas, y este fenómeno es un factor determinante para la modernización de los países en vías de desarrollo que, durante gran parte de su historia, renunciaron a tan valiosa contribución para la modernización de sus instituciones: «Hace tan sólo 50 años casi todas las mujeres marroquíes eran o amas de casa o esclavas. Casi todas eran analfabetas y se les prohibía salir de sus casas, salvo extraños casos. La tradición y los valores dominantes eran el pasto de todas las formas de pensamiento supersticioso y oscurantista. No tenían derecho a elegir a su futuro marido, los padres imponían sus elecciones y los hijos, hombres y mujeres, tenían que obedecer. Los matrimonios se formaban dentro de la tribu a menudo con el primo, dada la extensión de la familia patriarcal, la esposa se encontraba bajo la autoridad de su suegra, que decidía su destino. La poligamia era un fenómeno estructural» (Chafai, 1997: 43). Desde entonces, la realidad de la mujer marroquí ha cambiado mucho, y en esta línea la autora marroquí se pronuncia así acerca del cambio social producido a finales del siglo xx: "Con la expansión de la vida urbana y la descentralización del poder, la escolarización de los dos sexos, la participación masiva de la mujer en la vida pública y todas las manifestaciones de la vida moderna como la extensión de la familia nuclear, emergen nuevos valores y estructuras. El piso moderno extendido en todo el territorio urbano aparece como una revolución contra la casa tradicional [...] $»^{2}$. Aún así, Ramírez (2011) nos recuerda que la dominación masculina persiste y mantiene el velo entre sus instrumentos. Entre los complejos elementos culturales y religiosos que explican la persistencia del velo, prevalece el distintivo que separa a las «buenas musulmanas» de las «transgresoras». La mujer puede ser partícipe ahora de la vida pública e institucional, pero se le recuerda que

2. «[...] El nuevo espacio aparece concebido de tal manera que no puede admitir la presencia de una tercera persona (la suegra, la hermana divorciada, etc.). Inconscientemente la arquitectura refleja la futura familia, rechazando a la segunda mujer y etiquetándola como a un intruso. El nuevo espacio sólo permite una sola mujer, un hombre y algunos hijos (pocos)» (ibídem, p. 4). 
su existencia está justificada por la lealtad al grupo familiar, donde se impone el hombre. Asimismo, una visión religiosa de la vida y la sociedad condiciona enormemente la apertura femenina a nuevos ámbitos. Para Desrues (2009), la raíz del problema en el submundo islámico está en la enorme dificultad de «ejercer el derecho a ser no creyente», o sea, poder expresar libremente que no se confía en Dios, en el presidente o en el rey. Dicho submundo se estructura en torno a la familia y su patriarca, el varón. Asimismo, la tradición se convierte en la pretexto utilizado por los hombres para ejercer un cómodo dominio sobre sus mujeres, que, para Esteban de la Rosa (2009: 17), no responde a una lectura detenida fehaciente del Corán: «las revelaciones [del Profeta] no sitúan a la mujer en inferioridad respecto al hombre, sino que, por lo contrario, en la mayoría de las ocasiones, tratan de limitar el omnipotente y omnímodo poder del hombre en la sociedad pre-islámica, introduciendo la noción de protección a la mujer y de los-as niños-as, con un sentido de benevolencia hacia las personas más débiles de la sociedad».

Frente a estas barreras, la mujer desarrolla sus estrategias alternativas para ocupar nuevos espacios relacionales, aunando intereses y caminando hacia la modernidad (Newcomb, 2009). Esto lo logra fundamentalmente a través del contacto y el intercambio con otras mujeres, su emergencia en el trabajo fuera del hogar y la consecuente implicación en la formación más allá de la escuela primaria. En este sentido, es importante destacar que la mujer marroquí no estuvo nunca exenta de protagonismo y su contribución siempre fue importante. Su jornada de trabajo en el hogar ha sido maratoniana y ha estado, en muchas ocasiones, conectada a los negocios familiares. Belarbi (1991) la describió como el pilar de la economía marroquí, a pesar de estar bajo la etiqueta de papeles gregarios. "La mujer no presenta un discurso en el que se identifique como débil y secundaria en la vida familiar, ni se manifiesta como un ser dependiente que busque continua protección en el hombre. Ellas mismas se consideran un pilar para su familia. Sin embargo, las cosas cambian enormemente cuando nos preguntamos acerca de su proyección política y social. Aquí encontramos un espacio que incluso la propia mujer reserva para el hombre, [...] un precedente y una barrera difícil de superar, a pesar de las condiciones favorables que la modernidad ha facilitado a la mujer» (Vallejo Peña, 2010: 290). En definitiva, de una forma u otra, la mujer tuvo presencia en la gestión de los problemas sociales y en la toma de decisiones, aunque siempre desde la trastienda. Las marroquíes han sabido - a su modo- influir y condicionar los quehaceres diarios y la evolución de su sociedad.

En este sentido, Hernández Corrochano (2008b) nos destaca la dualidad vivida por el Marruecos de los años noventa con Hassan II en el poder. Mientras, a nivel institucional, se subrayaba la democratización de la sociedad marroquí, se realzaba, al mismo tiempo, el papel de la «buena familia»: extensa, patriarcal y unida, que considera a la mujer solo en su desempeño de roles familiares, menoscabando su individuación y su realización externa. A pesar de esto, la mujer expresa su satisfacción por su trabajo remunerado fuera de casa, aunque en su discurso prioriza siempre su papel en el hogar como esposa y madre. 
Abordaremos, por lo tanto, dos pilares esenciales dentro del proceso de emancipación de la mujer marroquí. Como primer pilar, la influencia del acceso femenino al trabajo remunerado sobre la nueva estructura social marroquí y, como segundo pilar, el acceso masivo de la mujer a la educación superior. Ambas circunstancias presentan dos facetas de un fenómeno social que ha actuado como ariete y elemento base para romper los moldes establecidos. No sólo ahora los hombres ven de otra forma a sus mujeres, sino que ellas mismas se ven como actoras sociales más activas y controladoras del cambio social. A partir de este punto, la mujer ha reivindicado su participación en el funcionamiento de las instituciones económicas y, además, gana independencia y poder de decisión ante el hombre. Esto se debe, fundamentalmente, a la recién adquirida capacidad para reunir recursos propios, así como las aportaciones dinerarias a la economía familiar (Vallejo Peña, 2010). Además, ahora la mujer ocupa nuevos roles a nivel social e institucional, gracias, entre otras cosas, a que puede construir, poco a poco, sus propias redes sociales.

En este sentido, es importante subrayar que la participación en la economía y el trabajo por parte de la mujer siempre se ha dado, procede de tiempo inmemorial. Siendo el hogar la histórica célula de la economía marroquí, la mujer siempre trabajó con más dureza que el hombre, aportando el sustento esencial del hogar y compaginando tareas tan dispares como la crianza de los hijos con el apoyo al negocio regentado por el marido (Mernissi, 1987) ${ }^{3}$. Dicho hogar actuaba como núcleo para la actividad femenina, pero, desde ahí, se ha ido proyectando gradualmente hacia el exterior. En este sentido, se pronuncia también el Informe de Desarrollo Humano (Naciones Unidas, 2006: 245) en sus conclusiones: «Lo impactante es que las mujeres marroquíes, no sólo están en una situación injusta de desigualdad, sino que lo están a pesar de desempeñar una función social y económica muy relevante, que podría resumirse en el hecho generalizado de ocuparse de la reproducción social y del cuidado de la familia, a la vez que son, con mucha frecuencia, el auténtico sostén económico de la familia, normalmente mediante el desarrollo de actividades económicas de subsistencia en la agricultura o la ganadería, la prestación de servicios o la confección o fabricación de manufacturas dentro de la economía sumergida, que en esta región (norte) tiene una fortísima presencia».

Entonces emerge con fuerza un factor determinante: la normalización del trabajo remunerado femenino fuera del hogar. Dentro de este proceso, la remuneración es un elemento esencial, tanto por su importancia para el mantenimiento económico del hogar, como por otras cuestiones sociales y psicológicas. Para Durán (2000), las sociedades patriarcales contrapusieron el empleo remunerado al trabajo en el hogar, y este último recayó en la mujer. Se trataba de dos formas distintas de generar valor social y económico. Ambas tenían un gran peso. Por tomar una referencia, la autora sugiere reflexionar sobre este trabajo no pagado en el caso de las mujeres en España, que supuso el 99\% del PIB español en 1996. Obviamente, estas cifras se disparan aún más

3. Edición original (1987). Se ha utilizado la edición revisada y en español (1999). 
en sociedades tradicionales patriarcales. La diferencia entre el valor generado por uno y otro está en que el empleo remunerado genera dinero flexible, que sirve de igual manera para pagar unas vacaciones, alimentos o un tratamiento médico, mientras que esto no es posible con el valor generado por el trabajo en el hogar. Este fenómeno contribuye a crear diferencias de estatus entre quien aporta el dinero flexible y quien aporta valor económico en otros términos. Por lo tanto, el salario se convierte en algo más que una simple dotación económica, adquiere un cariz simbólico como facilitador de la emancipación femenina (Alcalde, 2002). Ahora, la mujer puede llevar el timón de sus propios proyectos personales, con lo cual se evade de las redes en las que había quedado enmarañada en su dependencia del sueldo del varón.

Ahora bien, a pesar de la aportación dineraria al hogar, el trabajo remunerado entra en conflicto con la familia, que recuerda a la mujer su carácter nuclear en esta institución. En este sentido, Soledad Murillo (2007) afirma que, nada más comenzar a trabajar fuera de casa, la mujer debe gestionar su propia sustitución (la abuela, probablemente) y durante el desempeño experimentará una fuerte tensión entre roles. Estas presiones repercuten en una tasa de deserción laboral superior a la del hombre, ya que la propia mujer asume el predominio de la familia y de sus obligaciones como esposa y madre (Belarbi, 1995; Mernissi, 2007). Al mismo tiempo, la mujer sufre una fuerte discriminación salarial respecto al hombre en el mercado de trabajo. Esto está muy asentado en la costumbre, entre otras cosas, por la atribución otorgada al hombre como productor fuera del hogar. Belarbi (1997) afirma que esta discriminación está provocando, en países como Marruecos, que se emprendan cada vez más proyectos migratorios femeninos autónomos que persiguen al mismo tiempo un mejor salario, mejores condiciones de vida y reducir la presión social que la familia y el entorno vecinal ejercen sobre la mujer.

Para consolidar los logros de las mujeres en la estructura social y laboral marroquí, se ha contado con un segundo pilar: la formación, factor determinante en el mundo del trabajo. En la sociedad actual, el desarrollo económico de cualquier país exige la educación de sus mujeres, puesto que existe una estrecha relación entre el desarrollo económico y el aumento de las oportunidades educativas y laborales femeninas (Alberdi et al., 2000). En este sentido, es destacable el reciente fenómeno de la irrupción de la mujer marroquí en las universidades, con enorme pujanza en la última década, puesto que acapara ya más del 50\% de las plazas universitarias (Naciones Unidas, 2006). Otro fenómeno remarcable es la equiparación de ambos géneros recientemente también lograda en secundaria, con una tasa neta de asistencia del $36 \%$ en la mujer frente a un 39\% en los hombres (Unicef, periodo 2005-2009). En las aulas, buscan algo más que un título y una ubicación en el mercado laboral, gestan uno de los embriones del cambio. Mernissi ${ }^{4}$ observa positivamente este fenómeno en Marruecos y en el conjunto del mundo islámico, que contribuirá a mejorar las perspectivas de las mujeres y de los jóvenes en general. Frente

4. Op. cit., p. 7. 
a una globalización que trasmite a sus jóvenes mensajes agresivos, tanto de occidentales como de árabes, y un occidente que se niega a entender el mundo islámico desde los valores, la gran esperanza está en unos jóvenes que se forman con tasas de universitarios en países musulmanes superiores a las de otras naciones etiquetadas como "más modernas» y con una gran contribución de sus mujeres en estas cifras.

En estas nuevas condiciones, el hombre se ve invadido en territorios que ha dominado históricamente. La mujer asume roles de dominio y penetra, poco a poco, en el mundo laboral e institucional: jefas de servicios, plazas universitarias, así como puestos en los que puede desarrollarse como patrona. En el Marruecos actual, el 21\% de los funcionarios son mujeres y debemos considerar que se trata de cifras en aumento (Banco Mundial, 2005). Ella participa ahora financieramente y exige respeto y comprensión. El varón percibe la competencia de la mujer y debe cooperar con ella en ámbitos en los que no está acostumbrado a desenvolverse.

De cualquier forma, los notables progresos en el mundo educativo y laboral encuentran mayores barreras, al transformarse en representación institucional y activismo en la vida política y social (Soriano y Santos, 2002). Al respecto, en el señalado Informe AECID de 2007, se afirmaba entre las conclusiones (p. 212): «[...] la mujer marroquí no acaba de conciliar su rol de género con la actividad política, absolutamente arrastrada por la tradición. Se da una tendencia a ceder terreno en este tipo de responsabilidades al hombre. En algunas de las declaraciones recogidas, se percibe, incluso, un sentimiento de inferioridad respecto a los hombres en capacidades y habilidades para el desempeño en la vida política. A nuestro equipo se le ha creado la sensación de que el cambio de mentalidad en la clase media femenina marroquí está aún lejano». Asimismo, entre las mujeres marroquíes que han desempeñado cargos institucionales, se da un discurso de fuerte crítica hacia las barreras encontradas en la comunidad musulmana para desempeñar su representación de igual a igual. El hombre continúa ejerciendo un fuerte control sobre las relaciones sociales de la mujer, a quien le permite solo el contacto con hombres del entorno familiar y algunos amigos íntimos y cierra las puertas a otras relaciones externas, con la excepción de algunas amigas propias. En definitiva, el activismo político e institucional se presenta como un paso más complicado, aunque la consolidación laboral de la mujer contribuirá al progreso en este sentido.

Por otra parte, las dificultades expuestas en los ámbitos señalados (formación, empleo y participación social) se intensifican notoriamente en el ámbito rural, donde la mujer soporta la presión de una sociedad cerrada con un enorme peso del patriarcado. El impulso del cambio social en la mujer procede del entorno urbano y de sus clases medias y altas. La trasmisión de estos avances al medio rural conllevará un proceso temporal más amplio, así como un trabajo intenso ${ }^{5}$.

Asimismo, es importante resaltar que todo cambio social dirigido por los patrones que ha establecido durante el siglo xx el paradigma de la modernidad

5. Op. cit., p. 4. 
debe tener carácter endógeno. Al respecto, Solé (1997: 121) afirmaba: «[...] cada sociedad experimenta su propio proceso de modernización, de acuerdo con la existencia de condiciones concretas y agentes modernizantes propios, capaces de inducir o importar los cambios científicos, tecnológicos, sociales o políticos que desencadenan el proceso». Es decir, se deben evitar corrientes o tendencias que hagan a una sociedad caer en la artificialidad, buscando el cambio por el mero cambio, y bajo el anhelo de la «decorosa» etiqueta de la modernidad. La literatura analizada sobre el fenómeno sugiere que las mujeres marroquíes deben emprender y liderar su propio cambio, adaptado a sus pautas socioculturales y a las necesidades previamente experimentadas, así como asumiendo sus diferencias respecto a otros países que ya han pasado por el proceso de emancipación de la mujer.

\section{Objetivos y metodología}

El objetivo esencial de la investigación es valorar la reciente evolución de Marruecos y sus mujeres en materia de formación y empleo, así como las importantes repercusiones que los cambios en estos factores tienen en la estructura social del país. El análisis está planteado desde la perspectiva de valores y se afronta con un diseño multimétodo que explota, en una primera fase cualitativa, los resultados de los trabajos de campo anteriormente mencionados, realizados para la AECID en 2007 y 2008. En una segunda fase (cuantitativa), las pautas y conclusiones previamente establecidas se han contrastado con los datos obtenidos en las variables de la Encuesta Mundial de Valores (WVS) para Marruecos, más afines a las dimensiones objeto de estudio.

En la fase cualitativa, se han aplicado dos técnicas de investigación (ver cuadro 1): entrevistas en profundidad, realizadas a mujeres marroquíes de la región Tánger-Tetuán, así como un panel de expertos sobre estudios de género en Marruecos (hombres y mujeres) ${ }^{6}$. En ambos casos, se trató la evolución reciente de la mujer en la sociedad marroquí desde una perspectiva integral: religión, familia, trabajo, formación y participación política y social; si bien, durante las entrevistas y discusiones, se pudo observar un notable predominio de los discursos que incidían en el papel del trabajo y la formación en los nuevos roles de género de la mujer. Estas circunstancias incidieron en la elección de las variables y de las hipótesis que dan forma al presente estudio.

Las líneas de investigación y las conclusiones aportadas por las aplicaciones cualitativas invitaban a la obtención de un contraste cuantitativo (segunda fase). En esta etapa, el análisis se realiza desde una perspectiva longitudinal contrastando los datos obtenidos en Marruecos por el WVS de 2001 con los de 2007 (ver cuadro 2). En cada una de las variables seleccionadas, los datos

6. Desde ahora, las citas de informantes clave referenciadas como tales pertenecen al proyecto de $\mathrm{I}+\mathrm{D}$ desarrollado en Marruecos anteriormente señalado (apartado 1: «Planteamiento inicial»). Las citas textuales pertenecen a las entrevistas en profundidad, salvo los casos en los que se indica "panel de expertos». 
Cuadro 1. Fase cualitativa: datos técnicos

\section{Entrevista en profundidad}

Población objeto de estudio: mujeres entre 16 y 55 años de nacionalidad marroquí y residentes en la región Tánger-Tetuán. Este colectivo supone una cifra cercana a las 750.000 personas, sobre un total poblacional de 2.625.000 en la región.

Muestreo: no probabilístico y por cuotas.

Cuotas: los siguientes perfiles ocupacionales están representados con, al menos, dos entrevistas: estudiantes, empleadas de hogar, trabajadoras agrícolas, empleadas fabriles y del sector servicios, amas de casa (sus labores), profesionales liberales y empleadas públicas cualificadas. Asimismo, se han establecido tres cuotas de edad: de 16 a 29, de 30 a 44 y de 45 a 55 años, a cada una de ellas se ha asignado un tercio de la muestra.

$\mathrm{N}^{\circ}$ de entrevistas: 21.

$\mathrm{N}^{\circ}$ de preguntas: 58 .

Duración aproximada del contacto: 60-80 minutos.

Tipo: con guión semiestructurado y presencial.

Registro: grabadora digital.

\section{Panel de expertos}

Marruecos y sus mujeres; panel de expertos en estudios de género en Marruecos. Actividad incluida en el proyecto: TROYANO, J.F. (coord.) et al. (2008). La identidad de género de la mujer marroquí (II). Convocatoria PCI-AECID. Ministerio de Asuntos Exteriores.

Lugar y fecha de celebración: Facultad de Estudios Sociales y del Trabajo de la Universidad de Málaga, 25 de abril de 2008.

Perfiles de los participantes: investigadores del propio proyecto (2), investigadores externos de otras universidades implicados en estudios de género (3), imán de mezquita (1) y economista marroquí (1).

Duración aproximada (fase de conversación): 2 horas y 15 minutos.

Registro: grabadora digital.

Cuadro 2. Encuesta Mundial de Valores en Marruecos (2001, 2007): datos técnicos

Entrevista en profundidad

Población objeto de estudio: población marroquí mayor de 18 años (sin límite superior de edad). Muestra: 1.251 (1.247 ejecutadas) en 2001 y 1.200 en 2007.

Muestreo: estratificado (región y tamaño de la población) por cuotas (sexo y edad). Selección por rutas aleatorias.

Puntos de muestreo: 30 .

Error estimado: 2.

Limitación: sobrerrepresentación del medio urbano sobre el rural, corregido por ponderación.

Variables seleccionadas: V60: Ser ama de casa llena tanto como un trabajo remunerado (reacción ante la frase), V8: Importancia del trabajo en la vida, V48: Principales motivos para trabajar, V62: Importancia de ir a la universidad para hombres y mujeres (¿más para un chico que para una chica?) y V44: La consideración del empleo como un derecho preferente para los hombres respecto a las mujeres.

Las variables se han seleccionado por su vinculación a la dimensión sobre cambio social en empleo y formación en la mujer. Esta selección se ha visto condicionada por la repetición de las mismas preguntas en 2001 y 2007.

Fuente: Encuesta Mundial de Valores: Marruecos $(2001,2007)$. 
se presentan clasificados por géneros y tabulados. Las variables seleccionadas para su posterior análisis son: Ser ama de casa llena tanto como un trabajo remunerado (reacción ante la frase), Principales motivos para trabajar, Importancia del trabajo en la vida, Importancia de ir a la universidad para hombres y mujeres (¿más para un chico que para una chica?) y La consideración del empleo como un derecho preferente para los hombres respecto a las mujeres. Estas variables fueron identificadas y seleccionadas entre las ofertadas por la EMV por su capacidad para describir el fenómeno objeto de estudio: cambio social de la mujer en materia de formación y empleo.

Con nuestro análisis, pretendemos medir tanto la apertura en mentalidad como el incremento del interés de las mujeres marroquíes hacia la formación y el empleo remunerado. Ahora bien, la evolución del pensamiento masculino en las mismas dimensiones resulta igualmente trascendente, así como su posicionamiento respecto a la mujer (convergencia de los géneros o mantenimiento de la divergencia). No podemos olvidar que el movimiento de la mujer en Marruecos y en cualquier sitio pasa necesariamente por la implicación del hombre. Ellas deben actuar como motor, si bien ellos deben - gradualmente- secundarlas por el interés compartido de tener una mejor sociedad para sus hijos: hombres y mujeres.

A través de las previsiones obtenidas en la primera fase (cualitativa), se han establecido dos hipótesis previas. Mis previsiones apuntan a que el valor y la importancia que las mujeres dan en sus vidas al trabajo remunerado y a la formación respecto a otras cuestiones trascendentes ha aumentado entre 2001 y 2007 (hipótesis 1). En cuanto a los hombres, la expectativa se centra en que cedan más espacio a las mujeres en estos ámbitos, reduciéndose las diferencias en el estado de opinión respecto a la mujer en el transcurso de estos seis años (hipótesis 2). Desde ahora, denominaremos a la hipótesis 1 "consolidación», y a la hipótesis 2, «adaptación».

Por otra parte, al reflexionar sobre la situación de Marruecos y su sistema de valores, se debe destacar su posición en las dos dimensiones que Inglehart y Welzel (2005) consideraban esenciales para medir el grado de modernización de un país. En el eje vertical, aparece recogida la dimensión formada por el tradicionalismo y la secularización, mientras en el horizontal se presenta el eje formado por el materialismo y el postmaterialismo. Como se puede apreciar en la figura 1, Marruecos aparece con una «baja puntuación» en ambas dimensiones. El tradicionalismo y la secularización obtienen $-1,7$, y el materialismo y el postmaterialismo, $-1,2$. Estos datos sitúan a Marruecos en la zona inferior izquierda del plano, o sea, entre los países más distantes al modelo de la modernidad ${ }^{7}$.

La subdimensión sobre la participación laboral y formativa de la mujer marroquí sería indicativa de ambas dimensiones, aunque con particular acento en el materialismo y el postmaterialismo, tal y como destacan Inglehart y Welzel en sus planteamientos: «Aunque la emancipación femenina y su inte-

7. Op. cit., p. 5. 
Figura 1. Grado de modernización de países en función de su ubicación en plano bidimensional de Inglehart

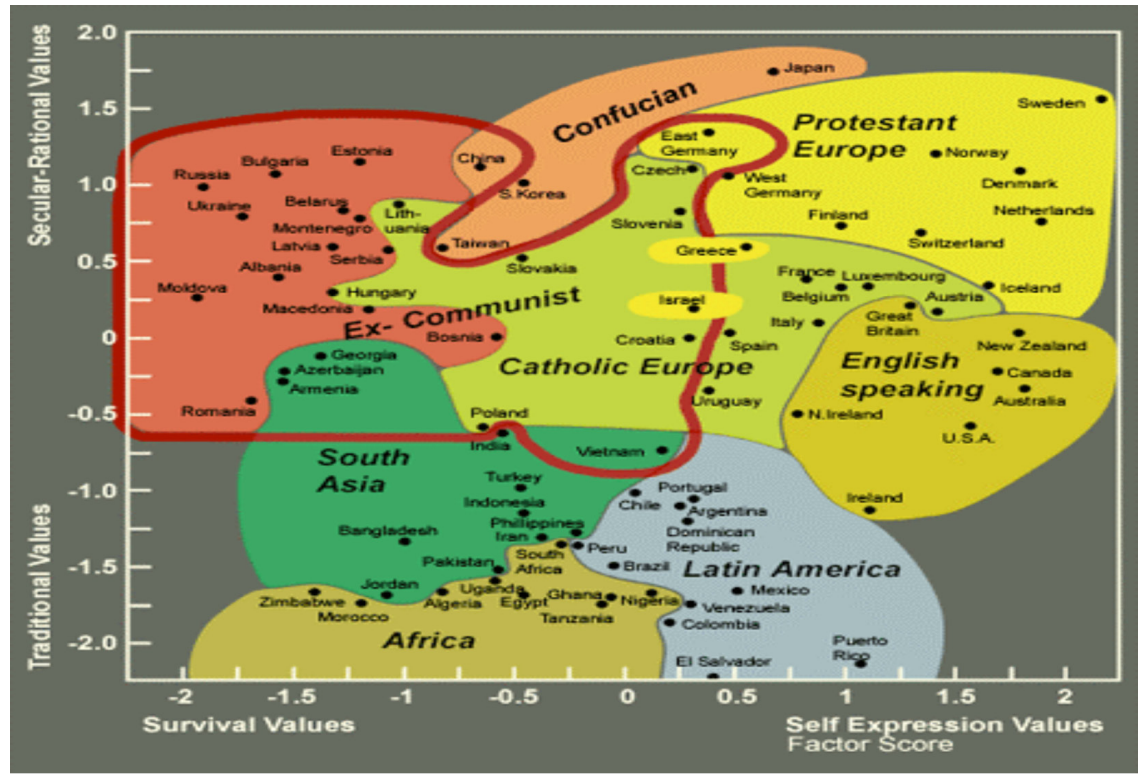

Fuente: INGLEhART, Ronald y Welzel, Christian (2005). Modernization, Cultural Change and Democracy. Nueva York: Cambridge University Press, 64. En base a datos de la Encuesta Mundial de Valores (www. worldvaluessurvey.org).

gración laboral son sólo algunas de las variables que determinan el acceso al postmaterialismo y a la modernización, también se presenta como síntoma de cambio en otras facetas (por ejemplo: la apertura de una sociedad a la participación de la mujer suele preceder también la participación de otros colectivos desfavorecidos como minorías étnicas, inmigrantes y homosexuales) ${ }^{8}$. Estas circunstancias refuerzan el peso de la variable sobre el conjunto de la dimensión (postmaterialismo) ${ }^{9}$. En caso de que la comprobación que se va a realizar a continuación delatara una mejora de las actitudes de las mujeres en el interés mostrado en desarrollarse en los ámbitos de la formación y el empleo (hipótesis de la consolidación); así como un acercamiento del varón a las mujeres en estas actitudes, aceptando en cierta medida la pertinencia del cambio (hipótesis de la adaptación), el pronóstico sería que Marruecos tenderá a desplazarse levemente

8. Ibídem, p. 12 .

9. El cambio de actitud de la mujer marroquí hacia el trabajo remunerado se presenta como un rasgo de postmaterialismo, ya que, en este caso, emergen causas que trascienden lo puramente económico (materialista). En ocasiones, un modesto salario obtenido fuera de casa no compensa demasiado las horas de servicio que la mujer deja de prestar en su hogar, sin embargo, para ella es importante por los factores que conlleva: un espacio social propio, autonomía, realización y participación. Se trata de elementos plenamente postmaterialistas. 
en la próxima década hacia el centro del plano en una trayectoria diagonal. $\mathrm{Al}$ menos si se verifican estas comprobaciones, se trazará esta hipótesis para investigaciones posteriores.

\section{Empleo y formación desde la perspectiva de la mujer marroquí: presentación de resultados}

A continuación, presentamos las variables anteriormente expuestas, tabuladas y segmentadas por sexo y fecha de aplicación de las preguntas (2001 y 2007). En el presente epígrafe, se analizan los resultados obtenidos en cada variable por separado, para posteriormente realizar un análisis conjunto en las conclusiones, así como su contraste con los resultados con la fase cualitativa. Todas ellas recogen cuestiones esenciales sobre la evolución de la percepción de hombres y mujeres de Marruecos sobre el empleo y la formación. De esta forma, se contrastarán las hipótesis planteadas en el estudio: (1) consolidación y (2) adaptación. El análisis ha sido dividido en tres líneas temáticas: trabajo doméstico frente a empleo remunerado, importancia del trabajo en la vida y motivaciones para desempeñarlo y, finalmente, derecho a la formación y al empleo.

\subsection{Trabajo doméstico frente a empleo remunerado}

La institución familiar ha condicionado tradicionalmente el desempeño femenino por su carácter absorbente. Dado el interés por analizar la consolidación del papel del empleo remunerado femenino en la sociedad marroquí como elemento de emancipación, resulta esencial comprobar si se reduce la presión de las obligaciones hogareñas sobre la mujer. Los resultados apuntan en esta dirección, puesto que se observan modificaciones sustanciales en la actitud de la población entre 2001 y 2007. Se observa como más de un tercio del conjunto de la población se mostraba "Totalmente de acuerdo» ante la afirmación «Ser ama de casa llena tanto como un trabajo remunerado» en 2001 (un 37,8\%), mientras que únicamente un 10,1\% estaba «Totalmente en desacuerdo». En tan sólo seis años la situación varía sustancialmente, ya que en 2007 es sólo el $25,9 \%$ sobre el total el porcentaje de personas que se muestran totalmente de acuerdo con la frase, mientras aumenta notablemente el nivel de desacuerdo (un 13,1\% en el conjunto y un 14,3 en las mujeres). Además, si agrupamos los porcentajes de las categorías «Totalmente en desacuerdo» y "En desacuerdo» encontramos que el 40,6\% sobre el total y el 44,2\% de las mujeres en 2007 muestra algún grado de disconformidad con la obligatoriedad del rol de ama de casa (en 2001 eran un 34,7\% y un 32,7\%, respectivamente). Esto refleja un incremento considerable en un plazo de tan sólo seis años (ver tabla 1).

Los discursos cualitativos analizados muestran la misma tendencia. La mujer corresponsabiliza ahora al marido de unas labores hogareñas que en un pasado reciente han estado circunscritas en todo momento al ámbito femenino. La mujer asume su papel nuclear en el hogar familiar, pero ahora pretende 
Tabla 1. Reacción de los entrevistados ante la afirmación «Ser ama de casa llena tanto como un trabajo remunerado»

\begin{tabular}{lcccccc}
\hline \multicolumn{1}{c}{ Opciones } & Total & Hombres & Mujeres & Total & Hombres & Mujeres \\
& 2001 & 2001 & 2001 & 2007 & 2007 & 2007 \\
\hline Totalmente de acuerdo & 37,3 & 35,7 & 38,8 & 25,0 & 24,1 & 25,9 \\
De acuerdo & 28,0 & 27,4 & 28,6 & 32,6 & 35,3 & 30,0 \\
En desacuerdo & 24,6 & 24,9 & 24,3 & 29,3 & 28,7 & 29,9 \\
Totalmente en desacuerdo & 10,1 & 11,9 & 8,4 & 13,1 & 11,9 & 14,3 \\
\hline Total & 2.098 & 1.025 & 1.073 & 1.192 & 589 & 603 \\
& $(100 \%)$ & & & $(100 \%)$ & $(100 \%)$ & $(100 \%)$ \\
\hline
\end{tabular}

Fuente: Encuesta Mundial de Valores (Marruecos: 2001, 2007).

abrir la puerta al desempeño de nuevos roles y, paso a paso, consigue arrastrar al hombre hacia un cambio gradual de mentalidad. Entre ellos, el $35 \%$ que defendía en 2001 que "Ser ama de casa llena tanto como un trabajo remunerado» ha quedado reducido en 2007 al 24,1\%. Estos hombres, presionados por los nuevos roles laborales de sus mujeres, comienzan ahora a entrar en un reparto de tareas que no hubiera sido admitido por la generación anterior. Estas circunstancias se reflejan en los discursos analizados:

Me costó un poquitín al principio con mi marido, porque él veía muy mal que el chico estuviese en la pila lavando los platos, [...] Cuando él llegaba y el chico tenía puesto el mandil, me preguntaba ¿por qué lo pones ahí? [se refiere al fregadero], yo no lo he puesto, él ha insistido en fregar y yo le he puesto a fregar ipor qué no? Que no, que él no es una chica, es un chico.

[...] Si estoy yo [en la cocina], él es muy cómodo, prefiere que cocine yo [risas], sí, aunque participe, pero muy raras veces. (Ama de casa con estudios primarios, 50 años)

Las marroquíes piensan inevitablemente en el comportamiento de género a sus hijos y transmiten sus nuevos valores a través de la educación. La división de tareas entre géneros en el hogar comienza a transmitirse como algo natural y necesario: ellos también pueden colaborar. La mujer podrá materializar el cambio de forma más efectiva sobre la generación posterior, entre otras cosas porque ahora los hijos comienzan a ver a su padre colaborando en labores domésticas que serán reproducidas por los varones adultos del futuro. El análisis cualitativo denota, entre las amas de casa marroquíes, las tensiones vividas en esta situación de transición.

¿Los hijos?... es obligación de los dos, y más si están trabajando los dos.

[...] Por supuesto que trabajaría aunque mi marido tuviera un sueldo suficiente. Por ejemplo, todos los meses doy dinero a mis padres. Ahora no voy a decirle a él que me dé para mandar a mis padres. No, no, no ¡eso no! A mí me da mucha dignidad. (Trabajadora del sector servicios con estudios primarios, 30 años) 
El cambio de mentalidad hacia el desempeño de los roles de género dentro y fuera del hogar se está produciendo tanto en hombres como en mujeres. Se han dado pasos importantes de cara a repartir funciones y responsabilidades de forma más equitativa. De hecho, un 67,6\% de los hombres reconocía estar «De acuerdo" o "Totalmente de acuerdo" ante la afirmación "Las mujeres deben contribuir también con ingresos al hogar» (según datos de la WVS2001). Las mujeres se pronunciaban así en un $81,1 \%$ de los casos. La no inclusión de esta variable en la encuesta de 2007 impide comprobar la evolución de estas actitudes, aunque la observación del fenómeno en su conjunto sugiere que el nivel de conformidad en este aspecto habrá aumentado aún más en los últimos años ${ }^{10}$.

Como consecuencia de lo anterior, ahora la mujer es más protagonista en su entorno social y exige un sitio en los procesos de toma de decisiones dentro y fuera del hogar. La posibilidad de que ambos obtengan ingresos fuera del hogar es también un estímulo para ellos y deben, por lo tanto, cooperar. Las presentes circunstancias están permitiendo que los hombres sean cada vez más permisivos a la hora de admitir la proyección de nuevos roles femeninos hacia el exterior, en aspectos sociales, laborales y formativos. Sin embargo, resultará mucho más complicado liberar a la mujer de las tradicionales obligaciones familiares, tan densas, que condicionan todo lo anterior. Todavía el compromiso con la institución nuclear de la sociedad marroquí y la religión islámica — la familia- dificulta y frena la emancipación de sus mujeres. Para ellas es difícil existir "por si solas», al margen del núcleo familiar, en el desempeño de un rol femenino carente de individuación (Hernández Corrochano, 2008a).

Es importante llamar a la reflexión sobre la importancia de estos cambios. Se da una atenuación gradual de lo que tradicionalmente ha sido una absorción integral de los roles femeninos por parte de la familia, de esta manera se abren las puerta hacia la formación, el empleo remunerado y la modernización de las mujeres marroquíes. La expansión del fenómeno ha llevado a algunas de ellas a apreciar la posibilidad de casarse con un extranjero no musulmán para abrir sus expectativas en formación y empleo para superar las dificultades señaladas (De Haas y Van Rooij, 2010). En este sentido, se está cimentando un cambio de mentalidad en la mujer marroquí, especialmente en las clases medias y altas y en ambientes urbanos, que busca nuevas vías de realización personal y construye para ello redes sociales, generalmente femeninas, que le aporten colaboraciones y un ambiente de seguridad y confianza en sus posibilidades ${ }^{11}$. Asimismo, es destacable el reciente acercamiento producido entre las actitudes de hombres y mujeres. Se trata de un factor que resulta esencial. Como destaca Kauffman (2007), los hombres son corresponsables de la integración.

10. Al no incluirse esta variable (2001) en la repetición del estudio en 2007 del WVS, en Marruecos se optó por no introducirla en el diseño de investigación entre los indicadores principales de este estudio, dada la ausencia de perspectiva longitudinal.

11. Op. cit., p. 4. 
En el proceso de apertura de la mujer, ellas deben ser el motor, pero el papel de los hombres como agentes facilitadores es esencial. El cambio no sólo está en las especulaciones, sino que también se manifiesta como algo real y es cada vez más tangible. De hecho, tanto los discursos cualitativos como los datos analizados en la encuesta corroboran las dos hipótesis planteadas: consolidación (1) y adaptación (2).

\subsection{Importancia del trabajo en la vida y motivaciones para desempeñarlo}

En cuanto a la importancia que el trabajo tiene en la vida para los marroquíes, debemos destacar que, desde el 2001, se muestran firmes en su consideración de "Muy importante». En esa fecha, los datos conjuntos indicaban un $89,4 \%$ en esta categoría, que ascienden a casi el total de la población si la agrupamos con la categoría "Bastante importante» (96,8\%). En 2007, las respuestas se mantienen en proporciones similares: un $85,8 \%$ señaló «Muy importante» y un $97,1 \%$ marcó una de las dos primeras categorías. Además, en 2007, tan sólo un 0,9\% del conjunto de la población marcó la categoría «Nada importante». En cuestiones de género no existen diferencias relevantes entre hombres y mujeres en esta variable. El equilibrio que presentan ambos grupos en estos valores delata una tendencia, en tiempos recientes, a la integración y asimilación de la clásica tesis de Jeremy Rifkin: el carácter central del trabajo en la vida (1996). La clave de este comportamiento está en que a los beneficios materiales del trabajo se fueron sumando otros no materiales (relación social, realización personal y profesional), que han calado también muy notoriamente en la mujer marroquí, que ve en el trabajo remunerado una alternativa para abrir su mundo y generar una mayor utilidad social, tanto para su entorno inmediato (familia) como para la ciudadanía en su conjunto.

El trabajo fuera del hogar se está convirtiendo también en un factor que, al margen de la realización profesional, está facilitando que la mujer consolide su identidad de género. Esto se debe a su liberación del rol de «leal servidora del hogar y de la institución familiar», una posición que no admite indivi-

Tabla 2. Importancia que los marroquíes dan al trabajo en la vida (marque una opción según la importancia que da al trabajo en la vida)

\begin{tabular}{lcccccc}
\hline \multicolumn{1}{c}{ Opciones } & Total & Hombres & Mujeres & Total & Hombres & Mujeres \\
& 2001 & 2001 & 2001 & 2007 & 2007 & 2007 \\
\hline Muy importante & 89,4 & 94,2 & 84,6 & 85,8 & 89,4 & 82,3 \\
Bastante importante & 7,4 & 4,9 & 9,9 & 11,3 & 8,6 & 13,9 \\
No demasiado importante & 2,1 & 0,4 & 3,7 & 2 & 1,4 & 2,6 \\
Nada importante & 1,1 & 0,4 & 1,8 & 0,9 & 0,7 & 1,2 \\
\hline Total & 2.250 & 1.116 & 1.134 & 1.197 & 592 & 605 \\
& $(100 \%)$ & $(100 \%)$ & $(100 \%)$ & $(100 \%)$ & $(100 \%)$ & $(100 \%)$ \\
\hline
\end{tabular}

Fuente: Encuesta Mundial de Valores (Marruecos: 2001, 2007). 
duación: se vive y se trabaja para el grupo. Corrochano ${ }^{12}$ denuncia la escasa contribución del Estado marroquí en su historia reciente que la mujer pueda ser considerada realmente un individuo, y no sólo en términos teóricos. En sus trabajos de campo en Tánger y Tetuán entre 1993 y 1997, la autora recoge la percepción de unas mujeres cansadas de existir sólo para crear y consolidar una buena familia. Por lo tanto, los espacios personales que libera el tiempo de trabajo no solo permite el desempeño de nuevos roles, sino que también abre las puertas a la realización personal al margen del núcleo familiar. Ellas comienzan a expresar la satisfacción que alcanzan al abrir estos nuevos itinerarios vitales:

Uno de mis sueños es acabar trabajando en una gran multinacional. [...] El trabajo fuera es algo muy positivo. La mujer adquiere nuevas experiencias fuera de casa y sale de la rutina que el hogar representa para ella. (Estudiante universitaria, 21 años)

Serenidad, responsabilidad, independencia... Es que me siento... iMe siento más fuerte que antes! Me ha cambiado mucho. En la imagen también, antes no me maquillaba... [Risas]. Aunque es una tontería, pero bueno [...]. Aquí, ahora, saco esa parte femenina mía. Sí, esto es un cambio positivo. (Trabajadora del sector servicios con estudios de secundaria, 30 años)

Ahora bien, el nuevo contexto de cambio en el desempeño de los roles de género femenino está abocado a encontrar una fuerte resistencia cultural. La generación actual de jóvenes mujeres trabajadoras en Marruecos afronta, en el día a día, frecuentes situaciones en su interacción social que le recuerdan el papel recesivo que le ha asignado el sistema tradicional patriarcal, y estas fricciones provocan algunas heridas en su orgullo personal:

Soy menos confiada con los hombres desde que trabajo y me enfrento a ellos. Resulta increíble que, para entrevistarme con un coordinador de proyecto, este exija un intermediario. (Representante de asociación y profesional liberal con estudios universitarios, 32 años)

$\mathrm{Al}$ analizar las motivaciones de los marroquíes para acceder al mercado de trabajo, destacaría el papel estelar del salario en el cambio de estilo de vida de las mujeres marroquíes. Esto se debe tanto a razones sociológicas como psicológicas. Observemos como en tan sólo 6 años la importancia del salario se duplica para el conjunto de la población: un 65,5\% en 2007 frente a un $31,3 \%$ (2001) marca la alternativa "Un buen salario» al preguntarse por el principal motivo para acceder al mercado de trabajo. Aunque ambos géneros le dan cada vez más importancia, el incremento es aún mayor en el caso de las mujeres (un 66,2\% en 2007, por un $33 \%$ en 2001). Además, es significativo el hecho de que este aumento se haya dado a costa de un valor tan apreciado como la seguridad del empleo. Sólo un $18 \%$ sobre el total marca «Un empleo 
Tabla 3. Motivaciones de los marroquíes para acceder al mercado de trabajo (Marque el motivo principal para buscar un empleo)

\begin{tabular}{|c|c|c|c|c|c|c|}
\hline Opciones & $\begin{array}{l}\text { Total } \\
2001\end{array}$ & $\begin{array}{c}\text { Hombres } \\
2001\end{array}$ & $\begin{array}{c}\text { Mujeres } \\
2001\end{array}$ & $\begin{array}{l}\text { Total } \\
2007\end{array}$ & $\begin{array}{c}\text { Hombres } \\
2007\end{array}$ & $\begin{array}{c}\text { Mujeres } \\
2007\end{array}$ \\
\hline Un buen salario & 31,3 & 29,6 & 33,0 & 65,5 & 64,8 & 66,2 \\
\hline Un empleo seguro & 44,2 & 45,4 & 43,0 & 18,0 & 18,8 & 17,2 \\
\hline Trabajar con gente que te agrade & 6,3 & 5,3 & 7,4 & 4,8 & 4,6 & 5,0 \\
\hline Realizar un buen trabajo & 18,3 & 19,8 & 16,7 & 11,8 & 11,8 & 11,7 \\
\hline Total & $\begin{array}{c}2.098 \\
(100 \%)\end{array}$ & 616 & $\begin{array}{c}589 \\
(100 \%)\end{array}$ & $\begin{array}{c}1.191 \\
(100 \%)\end{array}$ & $\begin{array}{c}591 \\
(100 \%)\end{array}$ & $\begin{array}{c}600 \\
(100 \%)\end{array}$ \\
\hline
\end{tabular}

Fuente: Encuesta Mundial de Valores (Marruecos: 2001, 2007).

seguro" como principal motivo de acceso al mercado de trabajo, mientras en 2001 era de un $44 \%$ (ver tabla 3 ).

Como se ha resaltado en el marco teórico, llevar un sueldo al hogar traído desde el exterior cambia notoriamente la estructura de las relaciones conyugales, puesto que se reducen las tradicionales relaciones de dependencia. La generación de recursos e ingresos desde dentro del hogar siempre fue una realidad palpable en el Marruecos tradicional, pero la introducción de esos ingresos externos, obtenidos por un reconocimiento social y laboral del entorno, cambia la psicología de estas relaciones.

Sí, por supuesto que he notado el cambio [desde que trabaja fuera de casa]. Hago uso personal de mi propio dinero. (Empleada de hogar sin estudios completados, 23 años)

Paso a paso, la mujer va exigiendo un espacio propio, para ejercer unas relaciones productivas y necesarias para el mantenimiento del hogar. Este fenómeno sirve de lanzadera de cara a facilitar la apertura de la participación política y social de la mujer, a fin de contribuir a fortalecer la estructura social y económica. Un trabajo por cuenta ajena puede abrir la puerta del autoempleo y de la creación de pequeñas empresas, y estos factores tendrán una gran repercusión en los nuevos roles que ocuparán las mujeres marroquíes. Además de esta proyección externa, las relaciones dentro del propio hogar cambian sustancialmente. Ahora las mujeres aportan ingresos, por lo que exigen tomar decisiones, entran en negociaciones más equilibradas que antes con sus maridos, los cuales, a su vez, están ahora más concienciados de la necesidad y la importancia del sueldo aportado por sus esposas. Ahora la mujer también contribuye a la economía doméstica con aportaciones en dinero flexible, el que sirve para adquirir cualquier bien o contratar cualquier servicio, lo que no es posible con las aportaciones en horas de trabajo. El trabajo en el hogar ha sido una aportación nuclear y esencial en la sociedad, pero algo que tradicionalmente la economía se ha negado a contemplar y resaltar. Estas labores se convierten en un tiempo ciego, que cae en el olvido, y queda como tiempo productivo contra 
tiempo no productivo. De hecho, en las sociedades tradicionales, el clásico salario masculino se ha fundamentado en el "no salario» femenino en el hogar y tiene su origen en este fenómeno, tal y como resaltaba Ángeles Durán ${ }^{13}$.

Asimismo, es importante resaltar que el trabajo para el hogar y la familia es esencial para cualquier mujer musulmana y esto está asumido e interiorizado. No se debe, por lo tanto, confundir la sensación de «no reconocimiento» —que es real- con la de «no realización». La modernización implica otras alternativas que deben facilitar y complementar su realización personal, si bien la aportación de las marroquíes a sus familias - en un gran esfuerzo por conciliar - reafirma el apego por su identidad cultural y práctica religiosa, que, obviamente, pretenden compatibilizar con sus nuevos roles.

[...] que yo vivo con mi religión y mis tradiciones estando muy orgullosa de ser musulmana, que empiezo a estar cansada de que francesas o españolas intenten convencerme de que para estar mejor tengo que parecerme a ellas. [...] Esto es algo más profundo, más complejo y tenemos que ser nosotras las que dirijamos nuestro propio cambio, a nuestro ritmo y a nuestra manera. (Economista marroquí: panel de expertos)

\subsection{Derecho a la formación y al empleo}

El cambio en las actitudes de la población a la hora de juzgar la pertinencia de que las jóvenes marroquíes vayan a la universidad marcha a fuerte ritmo. Tan sólo en el intervalo 2001-2007 se observa una drástica reducción entre aquellos que se mostraban completamente de acuerdo ante la afirmación «La universidad es más importante para un chico que para una chica» (ver tabla 4). Sobre el conjunto de la población, se ha pasado de un $25,3 \%$ a sólo un $14,8 \%$. Cifras de las que tiran las mujeres que, en esa misma categoría, han pasado de un $19,7 \%$ a un exiguo 3,6\%. Además, si agrupamos las categorías «En desacuerdo» y "Totalmente en desacuerdo», las mujeres muestran su rechazo en un $81,1 \%$ de los casos en 2007, bastante más que los hombres (un 59,4\%), que, sin embargo, han cambiado moderadamente su visión desde 2001 (un 50,5\%). Esta evolución en los valores está teniendo manifestaciones muy tangibles en la sociedad marroquí y en sus sistemas formativo y laboral. Estos datos respaldan las hipótesis de la consolidación (1) y de la adaptación (2). Los cambios que se están produciendo tienen ya consecuencias tangibles: el hecho de que 6 de cada 10 universitarios sean mujeres está generando nuevas perspectivas, dada la inevitable conexión de este mundo con la mano de obra más cualificada, tanto del sector público como del privado. Al margen de las cuestiones estrictamente laborales, la mujer adquiere un nuevo protagonismo en su entorno al completar la educación superior. Se trata de una demostración de su capacidad y de su preparación que avala a las marroquíes en su participación en el conjunto de las instituciones, incluida la propia familia. 
Tabla 4. Consideración de ir a la universidad como más importante para un chico que para una chica. (Exprese el grado de acuerdo con la frase: «Es más importante ir a la universidad para un chico que para una chica»)

\begin{tabular}{lcccccc}
\hline \multicolumn{1}{c}{ Opciones } & Total & Hombres & Mujeres & Total & Hombres & Mujeres \\
& 2001 & 2001 & 2001 & 2007 & 2007 & 2007 \\
\hline Totalmente de acuerdo & 25,3 & 30,8 & 19,7 & 9,2 & 14,8 & 3,6 \\
De acuerdo & 16,7 & 18,8 & 14,7 & 20,5 & 25,8 & 15,3 \\
En desacuerdo & 25,8 & 22,8 & 28,8 & 40,6 & 36,2 & 44,9 \\
Totalmente en desacuerdo & 32,2 & 27,7 & 36,8 & 29,8 & 23,2 & 36,2 \\
\hline Total & 2.067 & 1.038 & 1.029 & 1.146 & 569 & 577 \\
& $(100 \%)$ & $(100 \%)$ & $(100 \%)$ & $(100 \%)$ & $(100 \%)$ & $(100 \%)$ \\
\hline
\end{tabular}

Fuente: Encuesta Mundial de Valores (Marruecos: 2001, 2007).

Ahora bien, entre las tareas pendientes debemos destacar el carácter marcadamente urbano de este fenómeno en un Marruecos que cuenta con doce universidades para su extenso territorio y, al mismo tiempo, la notoria tendencia a la masificación del sistema: se trata de pocas universidades para un país con casi 32 millones de habitantes y una tasa de menores de 14 años del 27,8\% (Banco Mundial, 2010). Las clases más desfavorecidas de las grandes ciudades presentan dificultades de acceso y el problema se agudiza más aún en el ámbito rural, que continúa lejano al sistema universitario, tanto en el aspecto territorial como en la mentalidad.

En el medio rural, la mujer se ve frecuentemente envuelta en unas circunstancias opresivas en las que resulta difícil progresar a nivel personal por la carencia de recursos económicos y personales (formación), lo que, a su vez, imposibilita conseguir su independencia de los varones de su entorno familiar. De esta forma se dificulta la posibilidad de lograr superar las inseguridades de estas mujeres. En este sentido se pronuncia una de nuestras informantes:

Todavía hay miedo a la Administración, falta de confianza para enfrentarse a los entes: la policía, el asistente social, el juzgado... Para esto, además, el analfabetismo es una gran barrera [hablando del medio rural]. Cuando la mujer está formada y trabaja, aumentan sus opciones de representación. (Representante de asociación y profesional liberal con estudios universitarios, 32 años)

De Haas y Van Rooij ${ }^{14}$ destacan recientemente la movilización de mujeres rurales hacia ciudades universitarias para abrir su mundo. Sucede en las grandes ciudades de Marruecos y cada vez es más frecuente que estas chicas cursen sus estudios en universidades de Francia, España o Italia. Además de una formación académica y una capacitación profesional, ellas desean generar nuevas relaciones sociales y emprender proyectos personales, aprovechando la reduc-

14. Op. cit., p. 16. 
ción de la presión que ejerce sobre ellas su comunidad. Este fenómeno refuerza la tesis de las dificultades experimentadas por la mujer rural al tratar de abrir su ámbito formativo y laboral. En este sentido, Ramírez (1998) ya destacaba que, en la mayoría de los países europeos, estas mujeres pueden manifestar su práctica musulmana sin que esto conlleve cargar con la discriminación sexual de su país de origen. De esta forma se facilita la reconstrucción de su rol, al margen de las presiones familiares y vecinales.

El hecho de que se haya identificado, por parte de los dos autores precedentes, el desarrollo formativo de la mujer como factor que impulsa los proyectos migratorios de las más jóvenes, tanto a nivel interno como internacional, delata las dificultades experimentadas por las mujeres marroquíes en su desempeño diario. Si bien es importante especificar que estamos ante un Marruecos complejo y heterogéneo que hace difícil las generalizaciones sobre las circunstancias de las mujeres, tal y como trata de hacernos ver una de nuestras informantes.

Depende de que zonas, pueblos o ciudades. Tánger es un sitio más cercano a Europa [...] Pero hay zonas donde no se puede decir que esto es así. Yo lo que he visto me he quedado, de verdad..., impresionada. He visto mujeres, que, mientras que el hombre sale fuera a trabajar en el campo y la mujer queda con los niños, y en otro pueblo es todo lo contrario, la mujer se encarga de todo. No me lo esperaba: era una montaña enorme y las mujeres bajaban con los niños. Yo, cuando vi a las mujeres bajando con los hijos a su espalda, iban con los hijos a trabajar, eran las mujeres y no los hombres. Un pueblo del interior. Yo nunca había ido, a mí me impresionó. (Estudiante universitaria, 21 años)

En conexión directa con la percepción que los marroquíes tienen sobre el derecho de sus mujeres a ir a la universidad está la del derecho que tienen a un empleo, siempre en comparación con los hombres. La sociedad tradicional patriarcal concedió al hombre el rol del productor fuera del hogar y, según los resultados obtenidos en el WVS, se trata de una herencia que persiste. En 2001, las muestras de concienciación de la población marroquí sobre la prevalencia del empleo masculino sobre el femenino eran contundentes (ver tabla 5). Casi un $83 \%$ de los entrevistados se mostraba de acuerdo con la afirmación «Los hombres tienen más derecho a un empleo que las mujeres». Además, esta cifra no descendía demasiado al segmentarla por género, el respaldo femenino era de un $77,7 \%$. Sin embargo, observamos como el cambio producido en los datos sólo 6 años después (2007) es abrumador. El conjunto de los entrevistados ha reducido casi a la mitad su apoyo al derecho al trabajo del hombre con preferencia sobre la mujer (un $82,9 \%$ frente a un 50,8 ). El descenso se ha producido de manera aún más drástica si lo segmentamos por mujeres (un 77,7\% frente a un 36,7), mientras que casi la mitad de ellas (un 45,6\%) se manifiesta ya en desacuerdo con este viejo fundamento de la sociedad marroquí. Entre los hombres, debemos destacar que se ha pasado de un 7,2\% que se mostró en desacuerdo con la frase de referencia en 2001, a casi el triple de este porcentaje en 2007 (un 20,5\%). Esta variación ofrece interesantes muestras de cambio de mentalidad masculina, que presenta una variación significativa en el periodo 
Tabla 5. Conformidad con la frase «Los hombres tienen más derecho a un empleo que las mujeres"

\begin{tabular}{lcccccc}
\hline \multicolumn{1}{c}{ Opciones } & Total & Hombre & Mujer & Total & Hombre & Mujer \\
& 2001 & 2001 & 2001 & 2007 & 2007 & 2007 \\
\hline De acuerdo & 82,9 & 88,2 & 77,7 & 50,8 & 65,1 & 36,7 \\
Desacuerdo & 11,8 & 7,2 & 16,2 & 33,2 & 20,5 & 45,6 \\
No se pronuncia & 5,3 & 4,6 & 6,0 & 16,0 & 14,4 & 17,6 \\
\hline Total & 2.258 & 1.115 & 1.142 & 1.181 & 585 & 596 \\
& $(100 \%)$ & & & $(100 \%)$ & &
\end{tabular}

Fuente: Encuesta Mundial de Valores (Marruecos: 2001, 2007).

analizado, aunque todavía manifieste su defensa de una parcela históricamente adquirida y dominada. La colaboración masculina es necesaria en el proceso de integración institucional femenina en cualquier país y los datos manifiestan que la actitud de los hombres está cambiando (hipótesis de la adaptación). Asimismo, el conjunto de los datos analizados refleja una enorme aceleración en el cambio del sistema de valores sociopolíticos, al realizarse la recogida con un margen de tan sólo seis años (hipótesis de la consolidación). Además, ha aumentado significativamente el porcentaje de hombres y mujeres que optan por no pronunciarse sobre este asunto (de un 5,3\% a un $16 \%$ en los totales entre 2001 y 2007). Estas dudas en la población sugieren que su sistema de valores en cuestiones de género se encuentra en una situación de evolución y redefinición, factor que pudo haber aumentado la dificultad de la respuesta.

A pesar del paulatino pero constante cambio de mentalidad de la sociedad marroquí, otros factores deben acompañar y consolidar el fenómeno. El trabajo remunerado se está convirtiendo gradualmente en una cuestión normalizada y extendida entre ambos géneros, aunque costará mucho más que la mujer tome posiciones de liderazgo y con peso en la toma de decisiones en su desempeño. Estamos ante una sociedad en la que todavía la mujer percibe al hombre como líder político ideal en el 43,1\% de los casos, frente a un 73,7\% en los hombres (WVS-2007), y esta resistencia al reparto del protagonismo social y político dificultará el ascenso de la mujer en grado y sueldo. Además, la estructura del mercado laboral marroquí se presenta aún lejana a la situación de los países del primer mundo, en los que predomina el modelo de trabajo fuera del hogar para ambos cónyuges. Particularmente, resultará difícil generar puestos y cargos con cualificación media-alta suficientes para un país con cada vez más tituladas universitarias (un 38\% de tasa de matriculación neta en las mujeres por un $33 \%$ de los hombres en 2003, según Guía del Mundo, 2007). Esta adaptación requiere un complejo proceso de transición. Marruecos tardará en generar empleo suficiente para adaptarse a las futuras exigencias de la mujer, aunque en la actualidad ofrece muestras de auténtico cambio social y laboral con cifras muy destacadas entre los países del ámbito musulmán. En $2005^{15}$, más de 
10.000 mujeres realizaban ya funciones de patronas, es decir, empresarias que ofrecen empleo a hombres y otras mujeres.

En palabras de una de nuestras informantes: «Yo lo veo bien, han cambiado muchas cosas. Falta mucho por cambiar la verdad, por el tema del desarrollo, por el tema de los trabajos. Pero antes los hombres tenían muchos más derechos y hoy los cambios han favorecido a la mujer. Depende, si tú lo ves por política o democracia».

En líneas posteriores, continuando con el tema de los derechos de los hombres sobre las mujeres, nuestra colaboradora se pronuncia así:

[...] Antes, era el hombre el que tenía derecho a decir, a pedir el divorcio, que se le concedía por voluntad. Por ejemplo, si una mujer quería divorciarse, pero el hombre decía que no, pues la mujer tenía que aguantarse. ¡Pero hoy ha cambiado! [Refuerza esta afirmación elevando el tono de voz.] (Administrativa del sector servicios con estudios de secundaria, 29 años)

De cualquier forma, el cambio en el sistema laboral marroquí está en marcha, con las mujeres como ineludibles protagonistas. En un futuro inmediato, el aumento de la calificación de la mano de obra femenina (tanto por su presencia y su dominio de la formación secundaria como de la superior) facilitará su consolidación laboral. Los hombres y, en definitiva, la sociedad en su conjunto tendrá que asumir la necesidad de dar rienda suelta a un capital humano femenino capacitado, motivado y que fija su mirada más allá de los muros del hogar.

\section{Conclusiones}

En definitiva, el análisis realizado muestra un Marruecos en proceso de apertura a la modernidad y a la emancipación femenina, fenómenos que se manifiestan con logros significativos en materia de empleo y formación. Estos dos elementos están facilitando el desempeño de nuevos roles sociales en la mujer, que, gradualmente, supera su adscripción exclusiva a las tareas hogareñas y al servicio de la familia. Las protagonistas del cambio están experimentado una fuerte resistencia cultural que se acentúa en el mundo del trabajo remunerado, territorio que la sociedad marroquí había asignado al hombre. A pesar de las dificultades experimentadas por la mujer para consolidar su participación laboral, social y política, los efectos del cambio son ya muy tangibles. En este sentido se pronunciaba la síntesis del Informe de Desarrollo Humano (Naciones Unidas, 2006: 11): «la mujer marroquí ha tenido un papel importante en la evolución del potencial humano en el Marruecos independiente. Tras un periodo durante el cual la gran olvidada del proceso de desarrollo humano ha podido realizar, mediante un largo combate, avances que hoy en día se reconocen unánimemente. Prueba de ello es la reciente Reforma del Código de la Familia y el Código de Nacionalidad. Estos avances significan la coronación de la acción constante de un movimiento dinámico de mujeres, militante y perseverante». 
La literatura analizada en el presente trabajo subraya que los progresos de la mujer marroquí en empleo y formación conllevan dos elementos asociados fundamentales: primero, la generación de espacios de relación social propios, y, segundo, la percepción de un salario fuera del hogar que la sitúa en un nuevo término en la negociación familiar, en la que puede exigir participar en las decisiones y emprender sus propias actividades. En sus reivindicaciones, la mujer se asoma a través de estos canales pero aún debe dar el auténtico golpe de timón: llevar el cambio de forma más tangible a través de una participación política y social más activa. Si bien sobrepasar las barreras estructurales de las instituciones patriarcales, así como afrontar la resistencia al cambio del conjunto de su sociedad serán misiones complicadas.

$\mathrm{Al}$ realizar un acercamiento parcial, variable por variable, el estudio ofrece las siguientes valoraciones tras el análisis del WVS (2001-2007) y su triangulación con la fase cualitativa. La mayor parte de los indicadores seleccionados presentan resultados que confirman tanto la hipótesis 1 (consolidación) como la hipótesis 2 (adaptación). Se trata de las variables V60: Ser ama de casa llena tanto como un trabajo remunerado (reacción ante la frase), V48: Principales motivos para trabajar, V62: Importancia de ir a la universidad para hombres y mujeres (¿más para un chico que para una chica?) y V44: La consideración del empleo como un derecho preferente para los hombres respecto a las mujeres. Este no es el caso de la V8: Importancia del trabajo en la vida, que presenta unos resultados que no reflejan cambios en la opinión de hombres y mujeres en el periodo de referencia, de manera que sólo permite confirmar que el trabajo continúa siendo en 2007 un valor tan importante para ambos géneros como ya lo era en 2001 .

Por lo tanto, los resultados de la investigación muestran que el cambio social en las actitudes hacia la formación y el empleo de las mujeres y hombres de Marruecos se está produciendo realmente, de forma tangible, con reflejo en su sistema de valores, tal y como delata la observación del fenómeno, la visión de algunos expertos de la rama (ver marco teórico), el análisis de los discursos de los informantes y la variación de las respuestas de sus ciudadanos, reflejada en el WVS entre 2001 y 2007.

La mujer marroquí se ha visto encasillada, desde tiempo inmemorial, en roles de servicio a la institución familiar que evitaron su individuación. Desde que Marruecos y sus mujeres se encuentran en un notable proceso de modernización, ellas han dado importantes muestras de superación de los roles tradicionales y de apertura hacia otros nuevos que potencian su realización personal. En este proceso, ha desempeñado un papel fundamental su incorporación gradual al trabajo remunerado, que ha cambiado la estructura del propio hogar. El salario ha sido, en este sentido, una herramienta vital, tanto por cuestiones económicas como psicológicas. Por una parte, la mujer realiza aportaciones en metálico que sustentan las cuentas del hogar y, por otra, aumenta su capacidad de negociación y entra en la toma de decisiones. Paso a paso, los varones van asumiendo el nuevo reparto de roles, ya que contemplan los nuevos ingresos como necesarios, empiezan a colaborar con las mujeres en las tareas del hogar 
y a liberar espacios para que sus esposas desempeñen sus nuevas labores. De igual forma, ambos géneros van trasladando el nuevo modelo a la educación de los hijos. Además, la mujer ha sabido interpretar el importante papel que la formación debía desempeñar en su proceso de emancipación, y ya muestra una presencia superior al hombre en la enseñanza terciaria. Como consecuencia de todo lo anterior, Marruecos empieza a normalizar la presencia de mujeres en puestos de patrona (empresaria), mandos intermedios y labores técnicas, tanto en la Administración como en la empresa privada. A su vez, la presencia femenina en estos ámbitos abrirá sus perspectivas en participación social y política, con lo cual irá asumiendo poco a poco roles de mayor relevancia institucional. Desde estas nuevas posiciones, la mujer podrá activar más aún todo el proceso anterior.

A pesar de los datos positivos que se obtienen tras analizar este proceso de apertura de la mujer, las actrices nos revelan el sufrimiento de sus asperezas en el desempeño cotidiano. La resistencia cultural ofrecida por el entorno familiar y vecinal aún está presente, y a la mujer se le recuerda continuamente su origen y "vocación» hogareña y familiar, papeles en los que la circunscribe la educación musulmana que ha recibido. En su camino, todavía debe soportar dolorosas situaciones de discriminación social y laboral, tal y como reflejan los discursos analizados en la fase cualitativa. Asimismo, debemos asumir la naturaleza urbana y de clase media y alta del cambio, que se ve mucho más limitado en el ámbito rural, tanto en materia de empleo como de formación. Esta circunstancia está provocando cada vez más proyectos migratorios hacia grandes ciudades nacionales y extranjeras, aunque esto confirma la tenacidad mostrada por las mujeres marroquíes ante las adversidades.

Estos cambios en la situación social y laboral de la mujer marroquí son tan reales y efectivos que han tenido un claro reflejo en los datos demográficos del país. Brey (2009: 11) afirma que el índice sintético de fecundidad de Marruecos ha descendido súbitamente en los últimos tiempos, de modo que ha pasado de 7 hijos por mujer en 1970 a sólo 2,8 en 2005. Estos datos lo sitúan más cerca de las cifras del conjunto de países desarrollados $(1,7)$ que de sus vecinos africanos $(4,8)$. La modernización de Marruecos está en marcha y tendrá a la mujer en la vanguardia. Si retomamos el plano bidimensional de Inglehart, que compara el grado de modernización por países (ver la figura 1 en la página 12), observamos que Marruecos muestra una posición lejana a la modernidad en las dos grandes dimensiones analizadas ("tradicionalismo y secularización» y «materialismo y postmaterialismo»). Sin embargo, las muestras de cambio presentadas por Marruecos en un fenómeno tan determinante como la emancipación femenina en los años posteriores a la medición del autor (la matriz está generada con datos de 2001) sugieren que Marruecos se desplazará en breve hacia el centro del plano, con lo cual dibujará una línea diagonal. Por lo tanto, se pretende plantear esta hipótesis en investigaciones posteriores.

Finalmente, debo destacar que el análisis cualitativo de los discursos indica tan claramente la dirección que está tomando el cambio social de la mujer 
como el carácter particular, centrípeto y endógeno que condiciona el proceso. Ellas mismas, las mujeres de Marruecos, deben actuar como motor del proceso, en función de sus inquietudes y necesidades, apostando por cambios que faciliten el equilibrio entre la tradición musulmana en la que se han educado y los desafíos de la modernidad. En palabras de uno de nuestros informantes: «ellas tienen que hacerse a la idea de que tienen que liderar y movilizar su propio cambio, a su manera. Ellas lo visualizan mejor que nadie pero aún les falta iniciativa y organización. Fijémonos en cómo se ha dado el cambio en Occidente: con ellas al frente, tirando del carro» (imán de mezquita: panel de expertos). En definitiva, ellas tendrán que abanderar su propio cambio: a su estilo, a su manera y a su ritmo.

\section{Referencias bibliográficas}

Alberdi, Inés et al. (2000). Las mujeres jóvenes en España. Barcelona: Fundación La Caixa. Colección Estudios Sociales, 4.

AlCALDE, Rosa (2002). «Las mujeres marroquíes en Cataluña: entre la trasgresión y el cambio». Revista Catalana de Sociología, 18, 27-44.

Belarbi, Aisha (1991). Le salaire du madame. París: Le Fennec.

- (1995). Femmes rurales. Casablanca: Le Fennec.

- (1997). «Le mouvement associatif fémenin». Prologues, 9, 28-33.

Brey, Elisa (2009). «La transición demográfica en Marruecos». Documentos de Trabajo, $3(2), 1-27,11$.

Chafai, Laila (1997). «Las mujeres, sujeto de marginalización en Marruecos». Anales de Historia Contemporánea, 13, 35-55, 43.

De HaAs, Heinz y Van Roolj, Aleida (2010). «Migration as emantipation?: The impact of internal and international migration on the position of women left behind in rural Morocco». Oxford Development Studies, 38 (1), 43-62.

DESRUES, Thierry (2009). «El islamismo en el mundo árabe: Interpretaciones de algunas trayectorias políticas». Revista Internacional de Sociología, 65 (1), 9-28.

Durán, María Ángeles (2000). La contribución del trabajo remunerado a la economía española: alternativas metodológicas. Madrid: Instituto de la Mujer. Ministerio de Trabajo y Asuntos Sociales.

Esteban de la Rosa, Gloria et al. (2009). La nueva Mudawwana marroqui: Entre tradición y modernidad. Sevilla: Consejería de Empleo. Junta de Andalucía, 17.

Hernández Corrochano, Elena (2008a). «Diferentes perspectivas sobre el estudio de la familia en el norte urbano de Marruecos: Un análisis desde una perspectiva de género". Papeles del CEIC, 35 (1), 1-29.

- (2008b). Mujeres y familia en el Marruecos modernizado. Madrid: Cátedra.

IngLeHART, Ronald y WeLzel, Christian (2005). Modernization, cultural change and democracy. Nueva York: Cambridge University Press, 63-64.

Kauffman, Alicia (2007). Mujeres directivas: transición hacia la alta dirección. Madrid: Centro de Investigaciones Sociológicas, Opiniones y Actitudes, 56.

Mernissi, Fátima (1987). El harén político: el profeta y las mujeres. Madrid: Ediciones del Oriente y del Mediterráneo, 1999.

- (2007). Miedo a la modernidad. Madrid: Ediciones del Oriente y del Mediterráneo.

Murillo, Soledad (2007). "La Ley de Igualdad efectiva entre hombres y mujeres». Estudios de Derecho Judicial, 142, 95-110. 
Newcomb, Rachel (2009). Women of Fes: Ambiguities of urban life in Morocco. Filadelfia: University of Pennsylvania Press, 22.

Organización de las Naciones Unidas (2006). Cincuenta años de Desarrollo Humano. Perspectiva 2025, 245.

- (2006). Cincuenta años de Desarrollo Humano. Perspectiva 2025, 11. Síntesis para Marruecos.

Ramírez, Ángeles (1998). Migraciones, género e islam: Mujeres marroquies en España. Madrid: Agencia Española de Cooperación al Desarrollo.

- (2011). La trampa del velo. Madrid: La Catarata.

Rifkin, Jeremy (1996). El fin del trabajo: Nuevas tecnologías contra puestos de trabajo: el nacimiento de una nueva era. Barcelona: Paidós.

Solé I Puig, Carlota (1997). "Acerca de la modernización, la modernidad y el riesgo». Revista Española de Investigaciones Sociológicas (REIS), 80, 111-132, 120.

Soriano Miras, Rosa María y Santos Bailón, Carmen (2002). «El perfil social de la mujer inmigrante marroquí en España y su incidencia en la relación intercultural». Papeles de Geografía, 36, 171-184.

Troyano, José Fernando (coord.) et al. (2007). La identidad de género de la mujer marroquí. Programa: PCI Mediterráneo; AECID; Ministerio de Asuntos Exteriores. Entidades ejecutoras: Universidad de Málaga y Universidad Abdelmalek Essaadi.

- (2008). La identidad de género de la mujer marroquí (II). Programa: PCI Mediterráneo; AECID; Ministerio de Asuntos Exteriores. Entidades ejecutoras: Universidad de Málaga y Universidad Abdelmalek Essaadi.

Vallejo PeÑa, Francisco Alberto (2010). «Nuevas perspectivas en participación política social en la mujer marroquí». Revista Internacional de Pensamiento Político, 5, 287-306.

\section{Bibliografía web}

http://www.guiadelmundo.org.uy (edición de 2007, datos de Marruecos). [Consulta: 2 junio 2011].

http://www.worldvaluessurvey.org (datos de Marruecos, 2001 y 2007). [Consulta: 10 marzo - 14 diciembre 2010].

http://www.bancomundial.org (datos de Marruecos, 2005 y 2010). [Consulta: 23 junio 2010].

http://www.unicef.org/spanish/infobycountry/morocco_statistics.html (datos de Marruecos del periodo 2005-2009). [Consulta: 4 mayo 2011]. 